[SPECIAL EDITIONS on "NANO FIBER"-Review】

\title{
A Review of Dry Spun Carbon Nanotube Yarns and Their Potential Applications in Energy and Mechanical Devices
}

\author{
Yasuhiko Hayashi*1,\#, Yusuke Chiba*1, Hirotaka Inoue*1, \\ Masaki Hada*1,2, and Takeshi Nishikawa*1 \\ ${ }^{* 1}$ Graduate School of Natural Science and Technology, Okayama University, 700-8530 Okayama, Japan \\ ${ }^{* 2}$ Tsukuba Research Center for Interdisciplinary Materials Science, University of Tsukuba, 305-8573 Ibaraki, Japan
}

\begin{abstract}
Carbon nanotube (CNT) yarn drawn from CNT arrays has recently attracted considerable interest for various applications in wiring harnesses, composites, smart textiles, and high strength structural materials. Although the excellent properties and functions of CNTs are expressed at nanometer scale, it is quite difficult to maintain these nanoscale properties while upscaling to bulk CNT spun yarn. This paper aims to provide an overview of the rapid synthesis of spinnable tall and dense CNT arrays, the processing and improvement of the physical properties of CNT spun yarn, and the application of CNT spun yarn in the fields of energy and mechanical devices.
\end{abstract}

(Received 15 October, 2019; Accepted 14 January, 2020)

\section{Introduction}

Compared to individual isolated carbon nanotubes (CNTs), yarn-like macro scale assemblies of CNTs exhibit exceptional mechanical toughness, electrical conductivity, and resilience to bending stress. They have potential for an incredible range of applications in electronics, structural materials, and energy devices. CNT yarns can be integrated into textiles to create smart fabrics because of their unique properties such as their ultra-light weight, high aspect ratio, high electrical conductivity, high mechanical strength, and high thermal conductivity [1]. However, developing a technique that can preserve the intrinsic electrical and mechanical properties of individual CNTs remains a challenge during the upscaling processes from the nano- to macro-scale.

Owing to rapid research progress, CNT yarnbased fiber type electric double-layer capacitors (EDLCs) have become one of most promising energy storage technologies. It is now possible to integrate these devices into textiles to store harvested energy. The key material properties required for this application are a large surface area as well as high conductivity that can result in large specific capacitance [2].

Turning to mechanical applications, fiber actuators have attracted considerable attention as they are not only lightweight but also can generate high strain, allowing them to perform human mimetic motions, which are strongly demanded in the field of soft robotics [3]. Since coil-shaped soft-actuators fabricated from nylon-6, nylon-6,6, and polyethylene exhibited extremely high mechanical work per unit weight (1000 times higher than that of human muscle), the thermally induced contraction behaviors of polymer fiber soft-actuators have been well investigated in terms of long-term stability $[4,5]$. So far, a coil-shaped polymer thread was reeled with metalic wire as a heat source [4]. To avoid further increases in weight and the suppression of flexibility, CNT yarn is a good candidate to replace metal wires as heat sources.

This review focuses on recent progress in this field, including our works on tall and dense vertically aligned CNT array growth as well as CNT assembly into CNT yarns for energy and mechanical device applications.

\# corresponding author: Yasuhiko Hayashi (+81-86-251-8230, E-mail: hayashi.yasuhiko@okayama-u.ac.jp) 


\section{CNT arrays and CNT yarns}

The ability to continuously fabricate CNT yarns by dry spinning from a large-scale CNT forest represents an important step toward a wide range of potential applications. Typically, there are two types of CNT yarn production methods. One is the wet spinning method in which CNTs, especially short CNTs, are dispersed in polymer solutions, extruded, and spun into yarns [6,7]. The other is the dry spinning method in which CNT yarns are continuously made by pulling CNTs directly either from a CVD furnace [8] or from a tall and dense CNT array without using any binders. The second method is an efficient continuous method that produces CNT yarns with good properties [9-11].

\subsection{Synthesis and properties of spinnable CNT arrays}

Highly spinnable tall and dense vertically-aligned CNT (VA-CNT) arrays have been synthesized rapidly without water vapor on iron thin-films deposited on $\mathrm{Al}_{2} \mathrm{O}_{3} / \mathrm{Si}$ substrates using a thermal chemical deposition system (CVD) [12-14]. In the CVD process, the substrate is annealed at $350{ }^{\circ} \mathrm{C}$ under a hydrogen gas atmosphere to remove moisture and reduce the iron film. The deformation of the iron film into nanoparticles was induced at a temperature above $500{ }^{\circ} \mathrm{C}$. Then, acetylene gas was introduced as a carbon source at a synthesis temperature of 600 $700{ }^{\circ} \mathrm{C}$, initiating CNT growth that continued for $10 \mathrm{~min}$.

To ensure the high reproducibility of the dry spinnable tall and dense VA-CNT array substrates, the density and vertical alignment of the CNTs were controlled by optimizing the thickness of the iron catalyst film and the synthesis temperature.

The height and morphology of CNT arrays were characterized using a scanning electron microscope (SEM). The wall number and tube diameter of the CNTs were characterized based on high-resolution electron micrographs (100 samples were analyzed from each CNT array) obtained by the field-emission transmission electron microscope (TEM) at an acceleration voltage of $200 \mathrm{kV}$.

High-quality tall and dense vertically aligned rapidly-synthesized dry-spun CNT arrays were produced with the number of CNT walls predominantly varying from 2 to 5 with a selectivity of above $70 \%$, which are beneficial for practical bulkscale high strength carbon devices. In addition, the high stability and reproducibility of the water-vaporfree thermal CVD process ensured tube diameters in the range of 3 to $7 \mathrm{~nm}$ but below $10 \mathrm{~nm}$. Not only can we control the catalyst iron nanoparticle size to achieve a high areal density, but we can also fine-tune the CVD process conditions-specifically, the flow rate and duration (or residence time) of acetylene. The inlet temperature strongly affects the number of walls and tube diameters of CNTs in the arrays owing to hydrocarbon decomposition on catalyst nanoparticles.

\subsection{Fabrication and properties of CNT yarns}

Fig. 1 shows a CNT yarn that was spun directly from the VA-CNT arrays using a homemade servomotor on a slider system with rotation and slide speeds of $80 \mathrm{rpm}$ and $1000 \mathrm{~mm}$ per minute, respectively [15-17]. The twist angle is controllable by adjusting the rotation and slide speeds. Moreover, the twisting process provides shear force, which improves the CNT yarn's strength. Tensile strength measurements were performed using a tensile testing system. Electrical conductivity was measured at room temperature using the two-point measurement method.

The diameter and twisting angle of the CNT yarn were determined to be approximately $20 \mu \mathrm{m}$ and 20-25, respectively, using SEM. A tensile strength of $2 \mathrm{GPa}$ (CNT yarn gauge length of $25 \mathrm{~mm}$ ) was obtained (Young's modulus about $150 \mathrm{GPa}$ ). Although tensile testing conditions differ depending on the paper, the excellent strength of CNT yarn was obtained compared to the values of similar type of CNT yarns previously reported [18]. It should be noted here that the specific strength (the value obtained by dividing the tensile strength by the specific gravity) is $3850 \mathrm{kN}-\mathrm{m} / \mathrm{kg}$ for CNT twisted yarn with a diameter of $10 \mu \mathrm{m}$, which exceeds that of carbon fiber (for instance carbon fiber A4, $2457 \mathrm{kN}-\mathrm{m}$ / $\mathrm{kg})$ and Kevlar (2514 kN-m/kg) [19]. Moreover, it can surpass the specific strength of $3539 \mathrm{kN}-\mathrm{m} / \mathrm{kg}$ of highstrength and high-modulus carbon fiber. The electrical conductivity was $10^{6} \mathrm{~S} / \mathrm{m}$, but it is necessary to reach a conductivity above $10^{7} \mathrm{~S} / \mathrm{m}$ for practical use. Based on our measurements, it is indicated that pristine CNT fibers behave typically as semiconductors and have electrical conductivity less than the order of $10^{5} \mathrm{~S} / \mathrm{m}$ at room temperature. 
(a)
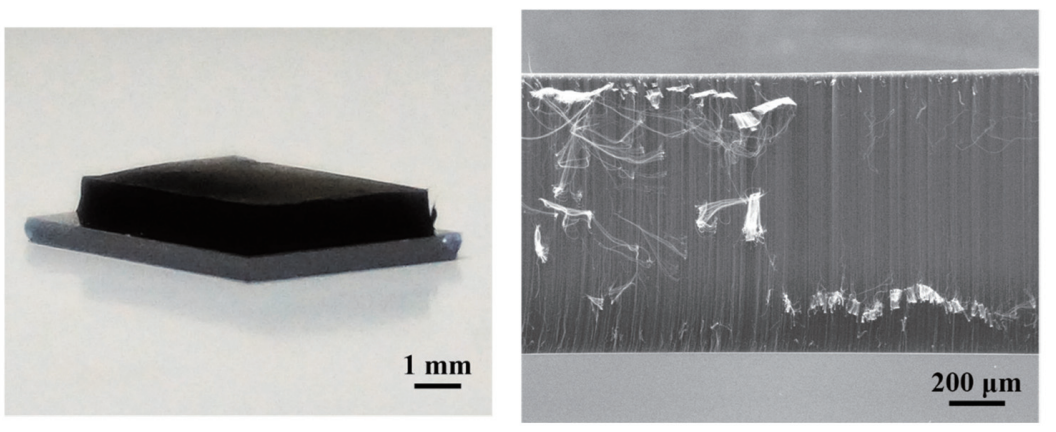

(b)

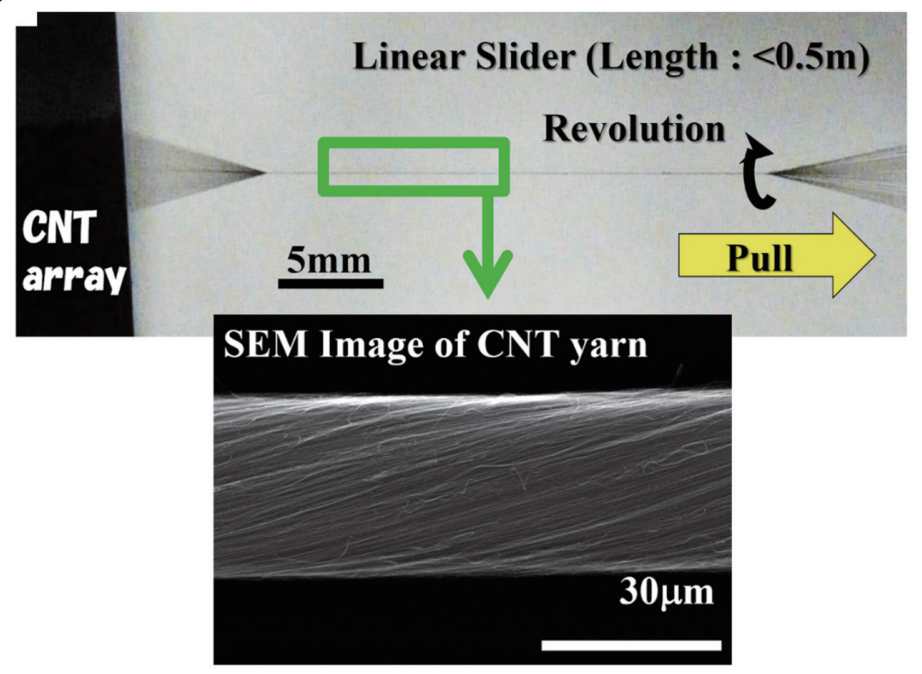

Fig. 1 (a) SEM image of the CNT array (left) and the CNT array ledge (right). (b) Spinning CNT yarn directly from tall and dense vertically aligned rapidly synthesized CNT arrays and typical SEM image of the CNT yarn.

\section{Applications in energy and mechanical devices}

Recent progress has elucidated many practical uses of these CNT yarns in developing ultralightweight and flexible energy and mechanical devices.

\subsection{Energy devices: CNT yarn-based fiber electric double-layer capacitors (EDLCs)}

We have demonstrated a simple architecture that can easily be scaled up to increase the EDLC length and significantly improve the electrochemical performance of the CNT yarn-based fiber EDLCs. First, each of the two CNT yarns (electrode materials) were soaked in a poly(vinyl alcohol)/phosphoric acid $\left(\mathrm{PVA} / \mathrm{H}_{3} \mathrm{PO}_{4}\right.$ ) electrolyte solution. After $24 \mathrm{~h}$ at room temperature, the sol electrolyte gelled. Then, the CNTs were weakly twisted without any separator material (two-ply yarn structure) to create a freestanding gel electrolyte-infilled CNT yarn-based fiber
EDLC and further dried at $60{ }^{\circ} \mathrm{C}$ for few hours, as shown in Fig. 2 (a).

The capacitive performance of the CNT yarnbased fiber EDLCs was measured through a twoelectrode system. Electrochemical impedance spectroscopy (EIS) measurements were performed in the frequency range from $1 \mathrm{~Hz}$ to $1 \mathrm{MHz}$ using a potentiostat.

Fig. 2 (b) shows the cyclic voltammetry (CV) curves of a CNT yarn fiber EDLC measured at different scanning rates. The $\mathrm{CV}$ curve of the CNT yarn EDLC nearly maintained a typical rectangular shape, even at a high scan rate of $0.2 \mathrm{Vs}^{-1}$, when the device was operated at scan rates ranging from 0.01 to $0.2 \mathrm{Vs}^{-1}$. Furthermore, it shows a minuscule equivalent series resistance of the CNT yarn, while the porous surface structure simultaneously induces rapid ion diffusion into the yarn [20]. The electrochemical capacitance was calculated as $3 \mathrm{~F} \mathrm{~g}^{-1}$ at a scan rate of $0.2 \mathrm{~V} \mathrm{~s}^{-1}$. The galvanostatic charge- 


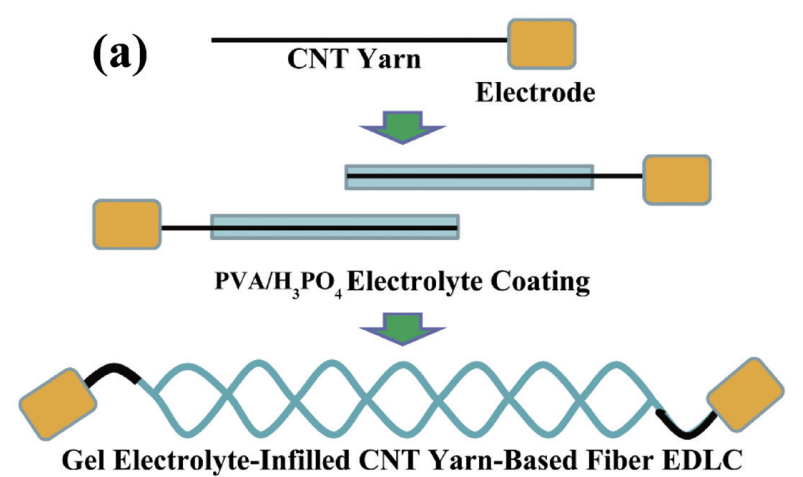

(b)

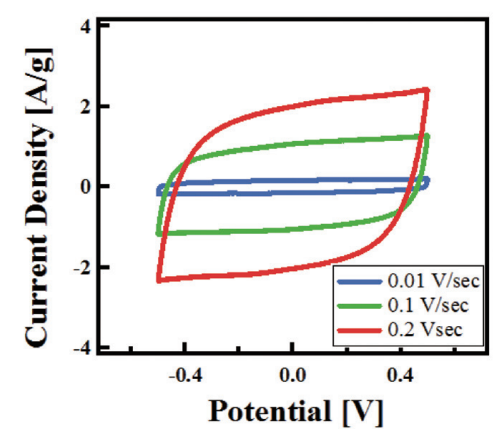

Fig. 2 (a) Schematic representation of the fabrication process of the CNT yarn-based fiber EDLC. (b) Cyclic voltammetry curves of a CNT yarn fiber EDLC at different scanning rates.

discharge (GCD) measurements of the pristine CNT yarn fiber EDLC were performed to evaluate the specific capacitance of the device at different current densities from 19 to $400 \mathrm{mAg}^{-1}$, as shown in Fig. 3. The plot shows an isosceles triangle and features a discharge curve that is almost linear with time, indicating good reversibility of the electrode reaction as well as superior EDLC capacitive behavior. The Ragone chart in Fig. 4 shows the power density with respect to the energy density of the flexible CNT yarn fiber EDLC. The highest values of power and

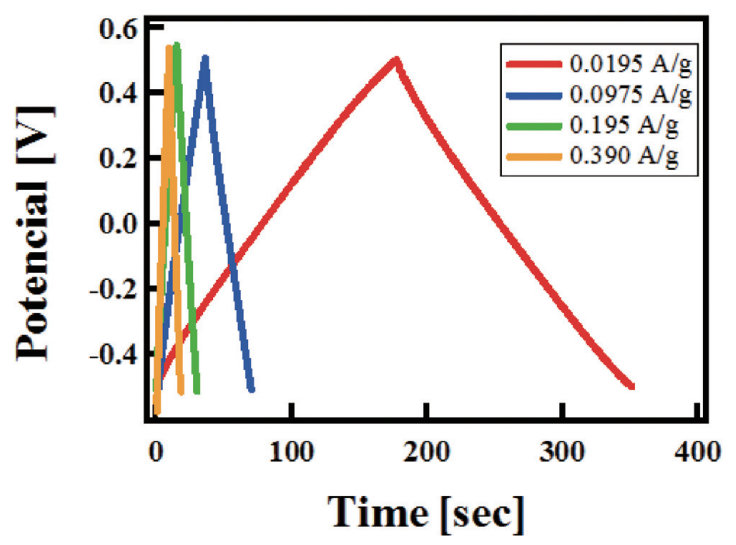

Fig. 3 Galvanostatic charge[discharge measurements of pristine CNT yarn fiber EDLC.

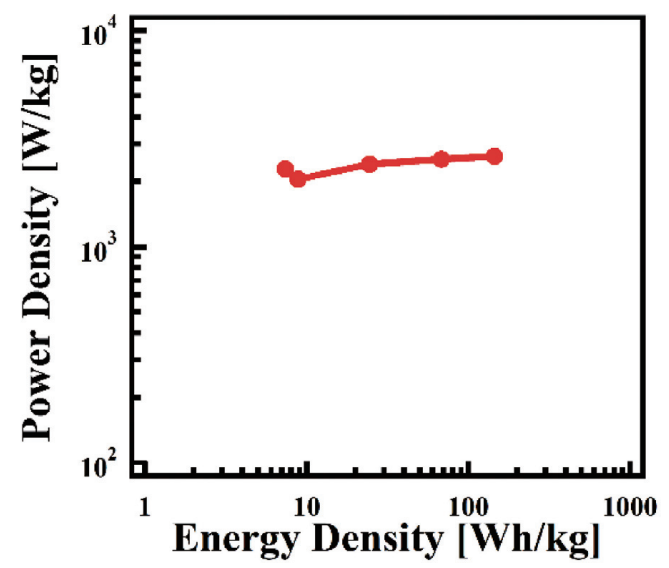

Fig. 4 Ragone chart of flexible CNT yarn fiber EDLC. energy density were found to be $2 \times 10^{3} \mathrm{~W} \mathrm{~kg}^{-1}$ and $100 \mathrm{Wh} \mathrm{kg}^{-1}$, respectively, and the device exhibited remarkably higher power and energy densities. The CNT yarn fiber EDLCs did not show any reduction in capacitance due to bending (approximately 45, 90 and 135 degrees, and knot); therefore, they possess superior mechanical stability and high flexibility and can be woven into textile structures.

\subsection{Mechanical devices: Coil-shaped polymer soft- actuators}

Pioneer work toward CNT based coil-shaped softactuators have been done by the group of Baughman et al. [21-23]. The basic actuation motion of a coiledshape soft-actuator composed of polymer or

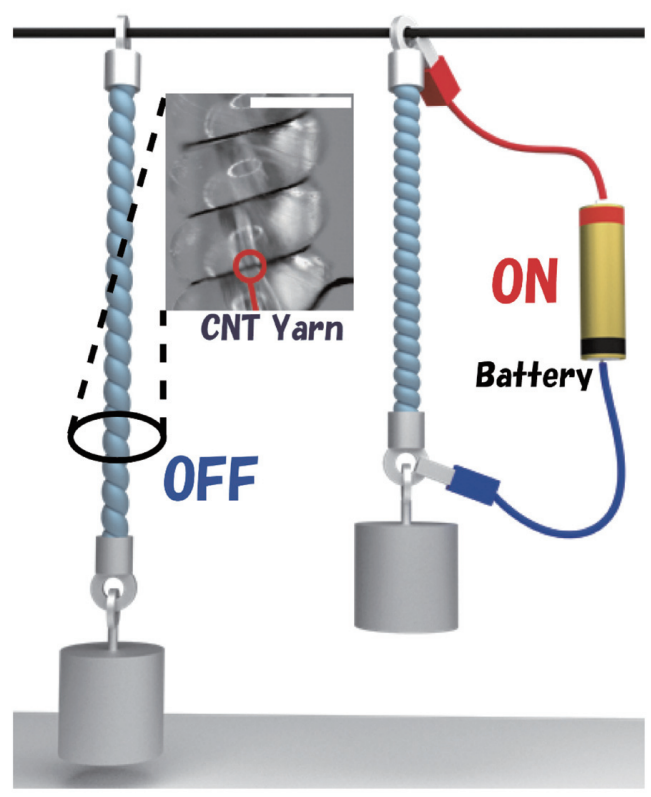

Fig. 5 Coil-shaped polymer soft-actuator composed of polymer threads and heat-introducing CNT yarns. The coiled polymer actuator generates large contractions by Joule heating under the battery connection. Thermally controlled reversible actuation is realized depending on a bias to CNT yarn. White scale bar, $500 \mu \mathrm{m}$. 


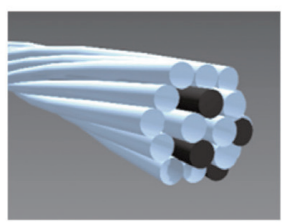

a)

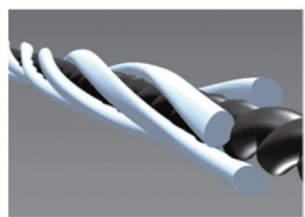

b)

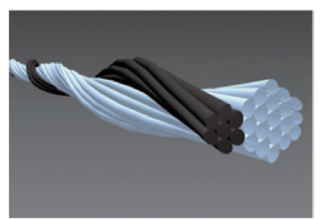

c)

Fig. 6 Schematic illustrations of soft actuators: a) multifilament-A (Multi-A),

b) multifilament-B (Multi-B), c) monofilament (Mono).

electroactive polymer thread or and metal wire is initiated by the thermally induced (via Joule heating of the metal wire) volume contraction of the polymer thread [24-26], similar to the one shown in Fig. 5. Problems remain regarding thermal distribution inside of the material and thermal dissipation from the soft-actuator, respectively. Moreover, the metal wire increases the weight and decreases the flexibility of the soft-actuator. To overcome these issues, we proposed and fabricated metal-free soft-actuators using three different structures of coiled shapes composed of poly(ethylene terephthalate) (PET) threads and heat-introducing CNT yarns [27]. PET is easy to use for soft-actuators because it has a glass transition temperature $\left(\mathrm{T}_{\mathrm{g}}\right)$ above room temperature and a high melting point of around $260{ }^{\circ} \mathrm{C}$. The lengths of the PET threads and CNT yarns were $15 \mathrm{~cm}$.

Fig. 6 shows schematic illustrations of the softactuators. Multifilament-A (Multi-A) is composed of a homogenous distribution of PET threads and CNT yarns. They were rotated together to form a coilshaped structure. Although it has a similar multifilament structure, multifilament-B (Multi-B) was formed using a slightly more complicated coil-shaping process. Initially, CNT yarns and four PET threads were bundled and rotated to fabricate coil-shaped PET/CNT fiber. Then, a certain number of coilshaped PET/CNT fibers were bundled together. To compare with multifilament structures, the monofilament (Mono), which is similar to the polymer thread and metal wire soft-actuator, was also fabricated from a bundle of PET threads and a bundle of CNT yarn strands.

The electric power applied to the CNT yarns was maintained at a constant $50 \mathrm{~mW}(5 \mathrm{~mW}$ per CNT yarn strand). The mechanical force linearly increased as the number of PET threads was increased in all types of fiber actuators. The mechanical force generated in Multi-A and Multi-B was 1.6 times higher than that generated in Mono, as shown in Fig. 7. The responsivities (heating and cooling processes) of the fiber actuator strongly depend on the time constant of

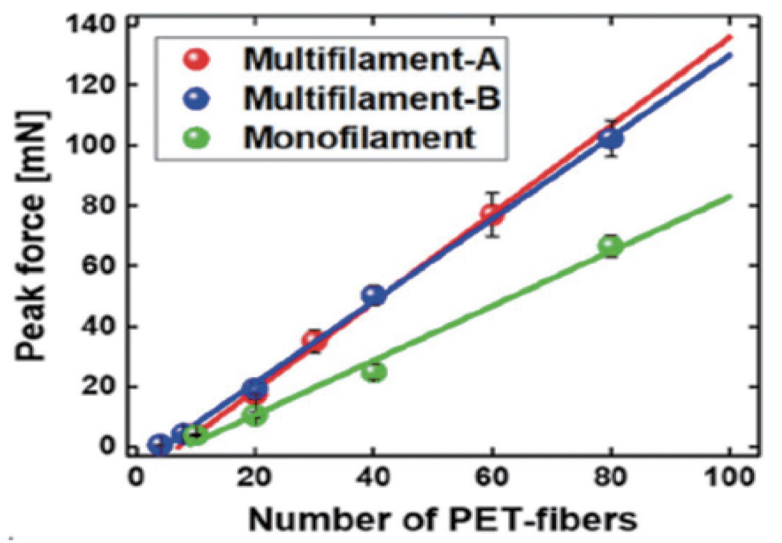

Fig. 7 Peak mechanical forces for soft-actuators as a function of the number of PET threads.

the deformation of the PET threads, and therefore the response behaviors of the three types of fiber actuators were identical. The amount of displacement of Multi-A and Multi-B was 1.4-1.8 times higher than that of Mono at $50 \mathrm{~mW}$, as shown in Fig. 8. This is in good agreement with the results obtained by the measurements of mechanical force.

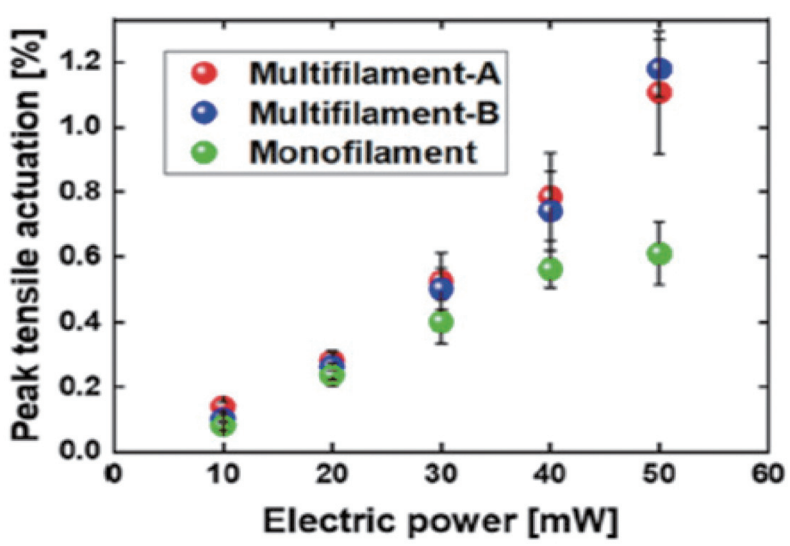

Fig. 8 Peak mechanical force for soft-actuators as a function of input electric power.

A more in-depth analysis of the thermal effects, thermal transfer, and temperature distribution in the fiber actuator was performed by constructing a model including both Multi-A and Mono using COMSOL Multiphysics $^{\circledR}$ [28]. Multi-A was homogeneously thermalized with the input energy, however, Mono showed an inhomogeneous thermal distribution. Based on our results, the homogenous thermal 
distribution in the fiber actuators realized by their brilliant structure is a key factor for achieving the highest actuation performance.

\section{Summary}

In this article, we comprehensively reviewed the synthesis of tall and dese vertically aligned CNT arrays, the preparation of CNT yarn spun directly from CNT arrays, and the applications of CNT yarns in various technologically important fields. CNT yarn and CNT yarn-based composite fibers have many advantages for application in energy storage or harvesting devices and soft mechanical actuators. EDLCs based on CNT yarn with excellent flexibility have great potential for electronic (or smart) textiles without sacrificing electrochemical performance. Twisted-coiled shaped soft-actuators consisting of polymer threads and CNT yarns might enable soft robotic devices without the need for mechanical motors because of their high actuation strain and work capacity. Although improving the performance of EDLCs and soft-actuators remains a challenge, these devices will soon become adequate for practical use.

\section{Acknowledgments}

The authors acknowledge financial support from the JSPS KAKENHI (Grant Numbers: 18H01708, 18K 18994, 19H05332 and 19K21946), the Saitama Industrial Promotion Public Corporation and Okayama Foundation for Science and Technology. We also thank Assistant Professor Wataru Takarada and Associate Professor Hidetoshi Matsumoto at the Tokyo Institute of Technology (Tokyo, Japan) for comprehensive support of experiments and discussions.

\section{References}

1. Z. Lu, Y. Chao, J. Foroughi, Y. Zhao, C. Wang, H. Long and G. G. Wallace, Nanoscale, 16, 5025 (2017).

2. Z. Lu, R. Raad, F. Safaei, J. Xi, Z. Liu and J. Foroughi, Front. Mater, 18, 1 (2019).

3. K. Y. Chun, S. H. Kim, M. K. Shin, C. H. Kwon, J. Park, Y. T. Kim, G. M. Spinks, M. D. Lima, C. S. Haines and R. H. Baughman, Nat. Commun., 5, 3322 (2014).

4. C. S. Haines, M. D. Lima, N. Li, G. M. Spinks, J.
Foroughi, J. D. W. Madden, S. H. Kim, S. Fang, M. Jung de Andrade, F. Göktepe, Ö. Göktepe, S. M. Mirvakili, S. Naficy, X. Lepró, J. Oh, M. E. Kozlov, S. J. Kim, X. Xu, B. J. Swedlove, G. G. Wallace and R. H. Baughman, Science, 343, 868 (2014).

5. A. Cherubini, G. Moretti, R. Vertechy and M. Fontana, AIP Adv., 5, 067158 (2015).

6. L. M. Ericson, H. Fan, H. Peng et al., Science, 305, 1447 (2004).

7. M. E. Kozlov, R. C. Capps, W. M. Sampson, H. Von Ebron, J. P. Ferraris and R. H. Baughman, $A d v$. Mater, 17, 614 (2005).

8. H. W. Zhu, C. L. Xu, D. H. Wu, B. Q. Wei, R. Vajtai and P. M. Ajayan, Science, 296, 884 (2002).

9. M. Motta, A. Moisala, I. A. Kinloch and A. H. Windle, Adv. Mater., 19, 3271 (2007).

10. M. Motta, Y. L. Li, I. Kinloch and A. Windle, Nano Lett., 5, 1529 (2005).

11. C. Jayasinghe, T. Amstutz, M. J. Schulz and V. Shanov, J. Nanomater., 2013, 309617 (2013).

12. T. Iijima, H. Oshima, Y. Hayashi, U. B. Suryavanshi, A. Hayashi and M. Tanemura, Phys. Status Solidi A, 208, 2332 (2011).

13. H. Matsumoto, S. Tsuruoka, Y. Hayashi, K. Abe, K. Hata, S. Zhang, Y. Saito, M. Aiba, T. Tokunaga, T. Iijima, T. Hayashi, H. Inoue and G. A. J. Amaratung, Carbon, 120, 358 (2017).

14. Y. Hayashi, K. P. Selvam and M. Scholz, "Rapid Growth of Dense and Long Carbon Nanotube Arrays and Its Application in Spinning Thread" in Carbon Nanotubes- Recent Progress, edited by Mohammed Rahman, InTech open access publisher, 118 (2018).

15. T. Iijima, H. Oshima, Y. Hayashi, U. B. Suryavanshi, A. Hayashi and M. Tanemura, Diam. Relat. Mater, 24, 158 (2012).

16. T. Iijima, Y. Inagaki, H. Oshima, T. Iwata, R. Sato, G. Kalita, T. Kuzumaki, Y. Hayashi and M. Tanemura, Mater. Express, 2, 357 (2012).

17. M. Scholz, Y. Hayashi, V. Khavrus, D. Chujo, H. Inoue, M. Hada, A. Leonhardt, B. Büchner and S. Hampel, Carbon, 133, 232 (2018).

18. S. N. Kanakaraj, N. T. Alvarez, S. Gbordzoe, M. S. Lucas., B. Maruyama, R. Noga, Y. Y. Hsieh and V. Shanov, Mater. Res. Express, 5, 065036 (2018).

19. S. Daggumati, "Concurrent Modeling and Experimetal Analyais of Meso-Scale Strain Fields and Damage in Woven Composites under Static and Fatigu Tensile Loading”, $\mathrm{PhD}$ Thesis; University Gent, 2011. 
20. Z. Lu, Y. Chao, Y. Ge, J. Foroughi, Y. Zhao, C. Wang, H. Long and G. G. Wallace, Nanoscale, 9, 5063 (2017).

21. R. H. Baughman, C. Cui, A. A. Zakhidov, Z. Iqbal, J. N. Barisci, G. M. Spinks, G. G. Wallace, A. Mazzoldi, D. D. Rossi, A. G. Rinzler, O. Jaschinski, S. Roth and M. Kertesz, Science, 284, 1340 (1999).

22. R. H. Baughman, Science, 308, 63 (2005).

23. C. S. Haines, N. Li, G. M. Spinks, A. E. Aliev, J. Di and R. H. Baughman, PNAS., 113, 11709 (2016).

24. C. S. Haines, M. D. Lima, N. Li, G. M. Spinks, J. Foroughi, J. D. W. Madden, S. H. Kim, S. Fang, M. Jung de Andrade, F. Göktepe, Ö. Göktepe, S. M. Mirvakili, S. Naficy, X. Lepró, J. Oh, M. E. Kozlov, S. J. Kim, X. Xu, B. J. Swedlove, G. G. Wallace and R. H. Baughman, Science, 343, 868 (2014).
25. M. Hiraoka, K. Nakamura, H. Arase, K. Asai, Y. Kaneko, S. W. John, K. Tagashira and A. Omote, Sci. Rep., 6, 36358 (2016).

26. J. H. Jeong, T. J. Mun, H. Kim, J. H. Moon, D. W. Lee, R. H. Baughman and S. J. Kim, Nanoscale Adv., 1, 965 (2019).

27. H. Inoue, T. Yoshiyama, M. Hada, D. Chujo, Y. Saito, T. Nishikawa, Y. Yamashita, W. Takarada, H. Matsumoto and Y. Hayashi, AIP Adv., 8, 075316 (2018).

28. COMSOL AB, Comsol Multiphysics: Structural Mechanics Module. COMSOL AB (Stockholm, Sweden, 2005). 